\title{
Cobalt protoporphyrin induces differentiation of monocytic THP-1 cells through regulation of cytoplasmic Ref-1-related NADPH oxidase activity
}

\author{
JU DONG SONG ${ }^{1 *}$, SANG KWON LEE ${ }^{2 *}$, SI EUN PARK ${ }^{1}$, KANG MI KIM ${ }^{1}$, \\ KOANHOI KIM ${ }^{3}$, YEONG MIN PARK ${ }^{1}$ and YOUNG CHUL PARK ${ }^{1}$ \\ ${ }^{1}$ Department of Microbiology and Immunology, Pusan National University School of Medicine; \\ ${ }^{2}$ Cardiovascular Center, Pusan National University Yangsan Hospital; ${ }^{3}$ Department of Pharmacology, \\ Pusan National University School of Medicine, Yangsan, Gyeongnam, Republic of Korea
}

Received April 28, 2011; Accepted June 3, 2011

DOI: 10.3892/ijmm.2011.736

\begin{abstract}
Cobalt protoporphyrin (CoPP) is a potent and effective metalloporphyrin inducer of heme oxygenase-1 (HO-1) activity in many tissues. Here, we report that CoPP induces differentiation of monocytic THP-1 cells into macrophage-like cells. CoPP induced a marked growth inhibition with a slight reduction in viability, and increased adhesion and spreading of THP-1 cells. However, other protoporphyrins did not. CoPP also resulted in expression of CD11b, MMP9, MSR1, CD14 and ICAM-1, which are differentiation markers for macrophages. Interestingly, we observed a decrease of cytoplasmic redox factor-1 (Ref-1) levels in the process of CoPP-induced differentiation of THP-1 cells. In addition, knockdown of Ref-1 by siRNA enhanced cell adhesion induced by CoPP. Furthermore, an inhibitor of NADPH oxidase, diphenyleneiodonium (DPI), completely abolished CoPP-induced adhesion of Ref-1-deficient cells using an siRNA. A cytosolic factor for NADPH oxidase activity, $\mathrm{p} 47^{\text {phox }}$, was significantly increased in THP-1 cells by CoPP treatment. Knockdown of Ref-1 increased CoPP-induced p47 $7^{\text {phox }}$ expression in THP-1 cells. Taken together, these results suggest that CoPP induces differentiation of monocytic THP-1 cells, and that the CoPP-induced differentiation is associated with cytoplasmic Ref-1-related NADPH oxidase activity.
\end{abstract}

\section{Introduction}

Reactive oxygen species (ROS) are recognized as important signaling mediators in the regulation of cell growth and differentiation. Intracellular redox status is greatly altered and

Correspondence to: Dr Young Chul Park, Department of Microbiology and Immunology, Pusan National University School of Medicine, Yangsan, Gyeongnam 626-870, Republic of Korea

E-mail: ycpark@pusan.ac.kr

${ }^{*}$ Contributed equally

Key words: cobalt protoporphyrin, THP-1 cells, differentiation, redox factor-1, NADPH oxidase becomes more pro-oxidizing in the process of differentiation of many types of cells $(1,2)$. Redox factor- 1 (Ref-1) is a ubiquitous multifunctional protein that acts as an essential regulator of intracellular redox status (3). Several studies have reported that stress signals lead to increased expression of Ref-1 and/or its nuclear translocation (4-6). In addition, decreased Ref-1 expression is associated with differentiation in many types of cells, including the retina, cardiac stem cells, and alveolar macrophages (6-8). However, the molecular mechanism underlying the role of Ref-1 in monocyte differentiation has not been elucidated.

Synthetic metalloporphyrins are known to be competitive inducers or inhibitors of heme oxygenase-1 (HO-1) activity in many tissues. Among them, cobalt protoporphyrin (CoPP) can act as the most potent metalloporphyrin inducer of $\mathrm{HO}-1$, which has cytoprotective activities and helps cellular homeostasis in response to stress and injury. Furthermore, CoPP is thought to be a promising therapeutic agent in cases in which HO-1 up-regulation is needed, without increasing oxidative stress (9), despite reported unexpected side effects (10-12). Until now, the effect of metalloporphyrin besides hemin on cellular differentiation has not been reported.

In the present study, we show that CoPP induces the differentiation of monocytic THP-1 cells into macrophage-like cells, in an NADPH oxidase-dependent fashion. We also show that CoPP-induced differentiation is associated with the expression of cytoplasmic Ref-1 in THP-1 cells.

\section{Materials and methods}

Reagents. CoPP, diphenyleneiodonium (DPI), protease inhibitor cocktail, Giemsa and trypan blue solutions were purchased from Sigma-Aldrich Chemical Co. (St. Louis, MO). Anti-Ref-1, HO-1, CD14, ICAM-1, p47 ${ }^{\text {phox }}$ and $\beta$-actin antibodies were purchased from Santa Cruz Biotechnology (Santa Cruz, CA). The secondary horseradish peroxidase (HRP)-conjugated antibody and the enhanced chemiluminescence (ECL) Western blotting kit were obtained from Amersham Pharmacia Biotech (Piscataway, NJ). Fetal bovine serum (FBS), HBSS, and other tissue culture reagents were purchased from Life Technologies (Gaithersburg, MD). 
Cell culture. The human monocytic leukemia cell line, THP-1, was grown in RPMI-1640 medium supplemented with $10 \%$ FBS, antibiotics (100 U/ml penicillin, $100 \mu \mathrm{g} / \mathrm{ml}$ streptomycin) and $10 \mathrm{mM}$ HEPES at $37^{\circ} \mathrm{C}$ in a humidified atmosphere containing $5 \% \mathrm{CO}_{2}$. The density of exponentially growing cells was maintained between $1-10 \times 10^{5}$ cells $/ \mathrm{cm}^{2}$.

Cell viability and growth analysis. THP-1 cells were plated at $5 \times 10^{5}$ cells per $60-\mathrm{mm}$ dishes. The cells were then cultured in various concentrations of $\mathrm{CoPP}$ in fresh medium containing $10 \%$ FBS and collected at $48 \mathrm{~h}$ after treatment with CoPP. Cells were resuspended in medium, and viable and dead cells were scored by the trypan blue dye exclusion method using a hemocytometer.

Adhesion assay. THP-1 cells were plated in 12-well tissue culture plates at $1 \times 10^{6}$ cells/well, and treated with various concentrations of CoPP for different periods of time. After incubation, the plate was washed 3 times with PBS. Adherent cells were fixed in methanol, stained with a $10 \%$ Giemsa solution, and measured by counting the number of cells in 10 randomly selected areas. For each sample, the total number of viable cells $[\mathrm{N}(\mathrm{t})]$ present at the indicated time points after chemical treatment and the sum of the number of adherent cells $[\mathrm{N}(\mathrm{a})]$ were determined to calculate the adherent ratio as follows: $[\mathrm{N}(\mathrm{a}) / \mathrm{N}(\mathrm{t})] \mathrm{x} 100$.

RNA isolation and RT-PCR. THP-1 cells $\left(1 \times 10^{6}\right)$ were grown in $60-\mathrm{mm}$ culture dishes, and incubated for $72 \mathrm{~h}$ in fresh medium with or without $100 \mu \mathrm{M}$ CoPP. Total-RNA was isolated using TRIzol reagent and reverse transcription was performed with superscript reverse transcriptase (Invitrogen, Carlsbad, CA) according to the manufacturer's instructions. Total-RNA $(2 \mu \mathrm{g})$ was used to prepare cDNA. The following primers were used in our study: CD11b, forward, 5'-CATAGCC AGCGGATAGCA-3' and reverse, 5'-GCAACTGTAGTTTC AGGGTC-3'; matrix metalloproteinase 9 (MMP9), forward, 5'-AGGACGGCAATGCTGATG-3' and reverse, 5'-AGGGCG AGGACCATAGAGG-3'; macrophage scavenger receptor 1 (MSR1), forward, 5'-GCAGTTCTCATCCCTCTCAT-3' and reverse, 5'-GGTATTCTCTTGGATTTTGCC-3'; glyceraldehyde 3-phosphate dehydrogenase (GAPDH), forward, 5'-TGAAGG TCGGAGTCAACGGATTTGGT-3' and reverse, 5'-CATG TGGGCCATGAGGTCCACCAC-3'. PCR reactions were performed in $25 \mu 1$ reaction volume with the following program: $95^{\circ} \mathrm{C}$ for $4 \mathrm{~min}, 30$ cycles of $94^{\circ} \mathrm{C}$ for $30 \mathrm{sec}, 60^{\circ} \mathrm{C}$ for $30 \mathrm{sec}, 72^{\circ} \mathrm{C}$ for $1 \mathrm{~min}, 72^{\circ} \mathrm{C}$ for $5 \mathrm{~min}$.

Preparation of cell and cytoplasmic extracts. At the end of the experiment, cells were washed with PBS and lysed in RIPA buffer with protease inhibitor cocktail to obtain total cell lysates. To prepare cytoplasmic extracts, cells were harvested, washed with PBS, resuspended in hypotonic lysis buffer (10 mM HEPES pH 7.9, $10 \mathrm{mM} \mathrm{KCl}, 1.5 \mathrm{mM} \mathrm{MgCl}_{2}$ ) supplemented with protease inhibitor cocktail, and incubated for $30 \mathrm{~min}$ at $4^{\circ} \mathrm{C}$. Cells were lysed by the addition of NP-40 (final concentration: 1\%). After $5 \mathrm{~min}$, the tubes were gently vortexed for $15 \mathrm{sec}$ and nuclei were removed by centrifugation at $500 \mathrm{x} \mathrm{g}$ for $5 \mathrm{~min}$. The supernatants were then quantified by the colorimetric method with bicinchoninic acid solution.

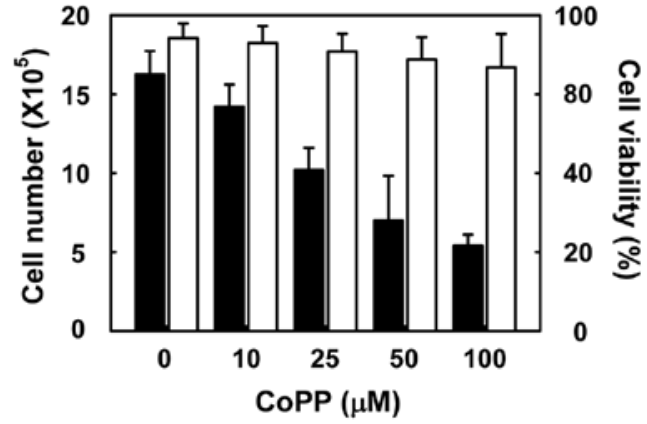

Figure 1. Effects of CoPP on cell viability and proliferation in monocytic THP-1 cells. Cell numbers ( $\square$, viable cells) and cell viability ( $\square$, viable cells/ total cells) were assessed by trypan blue dye exclusion assay at $48 \mathrm{~h}$ after CoPP treatment at the indicated concentrations in THP-1 cells. Values are the means \pm SD of 3 independent experiments.

Western blot analysis. Equivalent amounts $(10 \mu \mathrm{g})$ of protein were separated by $12 \%$ SDS-polyacrylamide gel electrophoresis. Proteins in the gels were transferred to nitrocellulose membranes using an electroblotting apparatus (Bio-Rad, Richmond, CA) and reacted with appropriate primary antibodies. Blots were then washed with TBS-T and incubated with HRP-conjugated secondary antibody. The membranes were developed with an ECL reaction kit and visualized using the LAS-3000 Luminescent Image Analyzer (Fujifilm, Tokyo, Japan). We used the Image Gauge version 3.0 software to calculate changes in protein expression. $\beta$-actin was used as an internal control.

Transfection of small interfering RNA (siRNA). A 21-nucleotide RNA duplex with 3'-dTdT overhangs was designed to interfere exclusively with the human Ref-1 mRNA (Dharmacon, Lafayette, $\mathrm{CO}$ ) in the 'ready-to-use' option. As a negative control, siRNA was also provided by Dharmacon. Ref-1 siRNA was introduced into THP-1 cells by electroporation using the Nucleofector (Amaxa Biosystems, Cologne, Germany), according to the manufacturer's recommended protocol.

Statistical analysis. Each experiment was repeated at least three times. Data were expressed as the means \pm SD from each independent experiment. The data for the experimental and control groups were evaluated for statistical significance by a one-tailed Student's t-test, with $\mathrm{P}<0.05$ accepted as the level of significance.

\section{Results}

Effects of CoPP on cell viability and proliferation in THP-1 cells. We first determined the effect of CoPP, a potent HO-1 inducer, on viability and proliferation of human monocytic THP-1 cells. Cells were treated with various concentrations of CoPP $(10,25,50$ and $100 \mu \mathrm{M})$ for $48 \mathrm{~h}$, and direct cell counts were determined by trypan blue dye exclusion. Cells did not exhibit significant reduction in cellular viability after $48 \mathrm{~h}$ with CoPP, even at high concentrations (Fig. 1). However, a significant decrease in cell numbers was obviously observed with dose-dependent concentrations of CoPP.

Effect of CoPP on THP-1 cell adhesion. We also examined the effect of CoPP on the adhesion of THP-1 cells onto tissue 


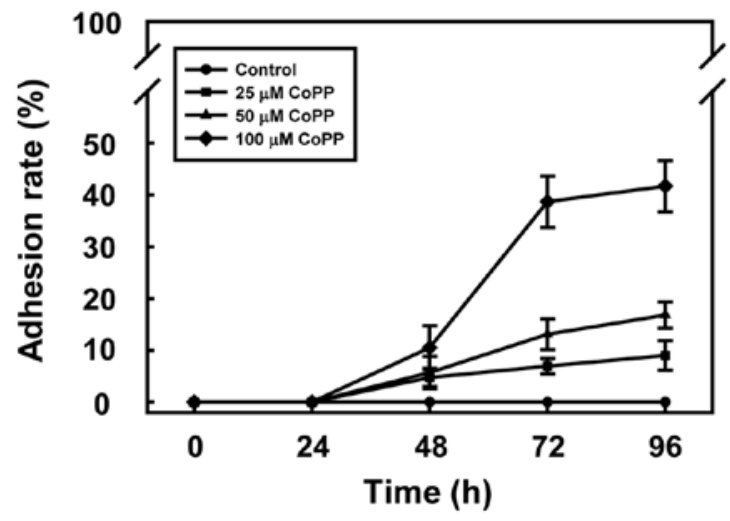

Figure 2. Effect of CoPP on cell adhesion. THP-1 cells were treated with different concentrations of CoPP for 24, 48, 72 and $96 \mathrm{~h}$. Data shown represent relative cell adhesion. Three independent experiments were performed and values are the means $\pm \mathrm{SD}$ obtained from triplicate experiments.
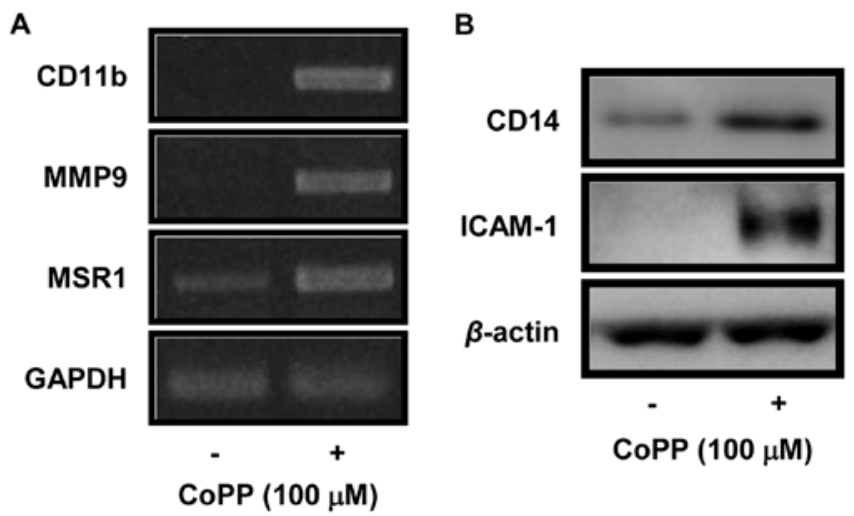

Figure 3. Effect of CoPP on the expression of macrophage differentiation markers. THP-1 cells were incubated with or without $100 \mu \mathrm{M} \mathrm{CoPP}$ for $72 \mathrm{~h}$. (A) mRNA levels of CD11b, MMP9 and MSR1 were analyzed by RT-PCR. GAPDH was used as an internal control. (B) Cell lysates were analyzed by Western blot analysis to detect expression levels of CD14 and ICAM-1. $\beta$-actin was used as an internal control to monitor equal protein loading.

culture plates. Cells were treated with various concentrations of CoPP $(25,50$ and $100 \mu \mathrm{M})$ for $24,48,72$ and $96 \mathrm{~h}$, as indicated. Adhesion of THP-1 cells on plates was significantly increased at $72 \mathrm{~h}$ after CoPP treatment (Fig. 2). Control cells did not adhere to the culture plate and maintained their round shape during this experiment. Interestingly, in contrast to CoPP, other metalloporphyrins, hemin, SnPP and ZnPP, did not have any effects on adhesion and morphology related with differentiation of THP-1 cells (data not shown).

Effect of CoPP on the expression of differentiation markers in $T H P-1$ cells. Since monocyte differentiation is associated with the expression of surface proteins required for macrophage functions, we next analyzed the expression of differentiation markers, such as CD11b, MMP9, MSR1, CD14 and ICAM-1, after treatment with CoPP. In unstimulated cells, CD11b, MMP9 and MSR1 mRNA were either not detected or weakly detected. However, the expression of those mRNAs was significantly increased by CoPP treatment in THP-1 cells (Fig. 3A). Similarly, the levels of CD14 and ICAM-1 proteins were also strongly induced by CoPP treatment (Fig. 3B).

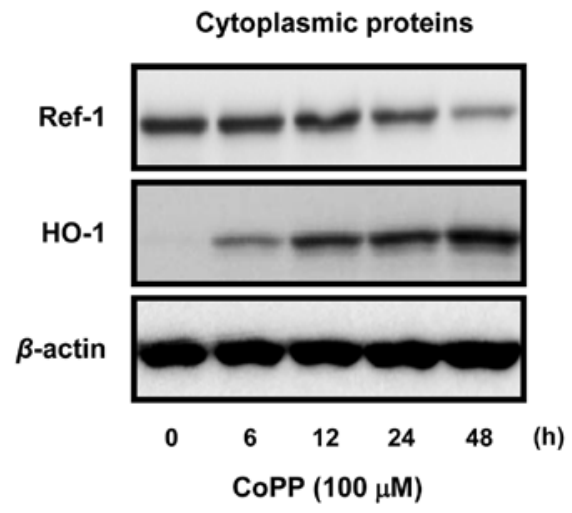

Figure 4. Involvement of Ref-1 in CoPP-induced differentiation. THP-1 cells were incubated with $100 \mu \mathrm{M}$ CoPP for up to $48 \mathrm{~h}$. At the indicated time points, cytoplasmic proteins were isolated and analyzed by Western blot analysis using antibodies for Ref-1 and HO-1. $\beta$-actin was used as an internal control for cytoplasmic proteins.

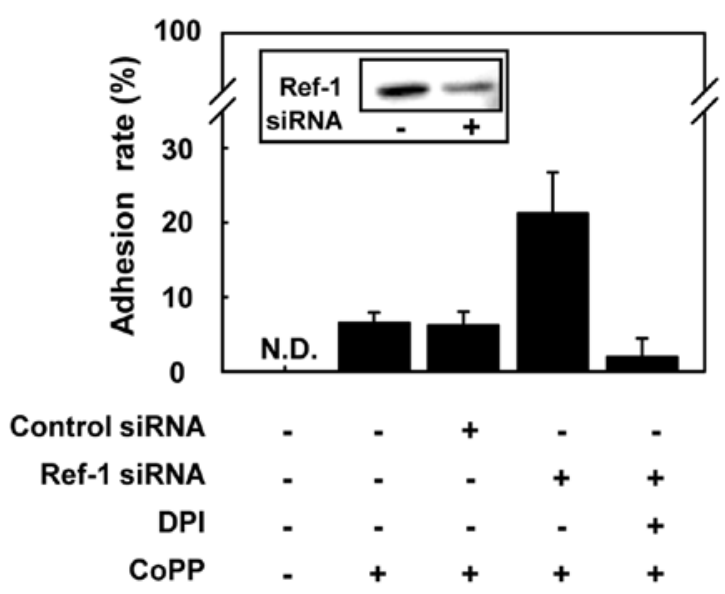

Figure 5. Effect of Ref-1 siRNA on CoPP-induced adhesion of THP-1 cells. Cells were transfected with siRNA for silencing of the Ref-1 gene. The cells were then pretreated with $5 \mu \mathrm{M}$ DPI for $30 \mathrm{~min}$ and incubated with $50 \mu \mathrm{M}$ CoPP for $48 \mathrm{~h}$. Cell adhesion was analyzed. The inset shows the efficiency of Ref- 1 silencing. Values are the means \pm SD of 3 independent experiments.

Involvement of Ref-1 in THP-1 cell differentiation by CoPP. We next examined the role of Ref- 1 , a regulator of the intracellular redox status, in the CoPP-induced differentiation of THP-1 cells. As expected, CoPP resulted in a significant increase of HO-1 expression in THP-1 cells. However, a dramatic loss of cytoplasmic Ref-1 was observed at 24 and $48 \mathrm{~h}$ following treatment of CoPP (Fig. 4). Therefore, we examined whether the knockdown of Ref-1 affects CoPP-induced differentiation of THP-1 cells. For this experiment, we used oligonucleotides interfering with Ref-1 mRNA (siRNA) for the inhibition of Ref-1 expression. siRNA efficiently silenced the Ref- 1 gene and completely suppressed Ref-1 levels (Fig. 5). Importantly, knockdown of Ref-1 significantly increased cell adhesion induced by CoPP. Ref-1 siRNA alone had no effect on adhesion of THP-1 cells. Furthermore, an inhibitor of NADPH oxidase, DPI, completely abolished CoPP-induced adhesion of THP-I cells.

Correlation of Ref- 1 and NADPH oxidase in CoPP-induced THP-1 cell differentiation. ROS driven by NADPH oxidase plays an important part in cell proliferation, differentiation, and 
A

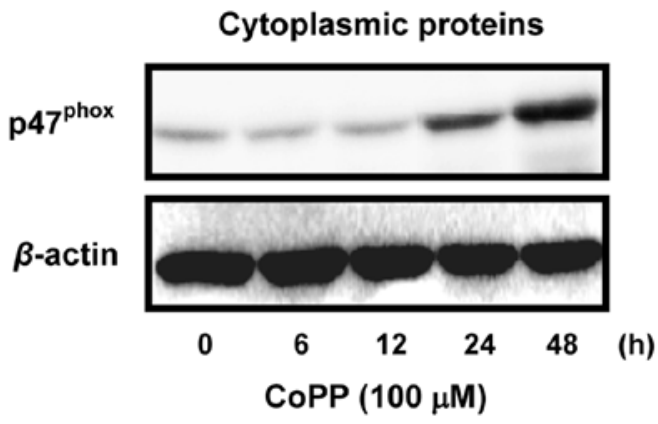

B

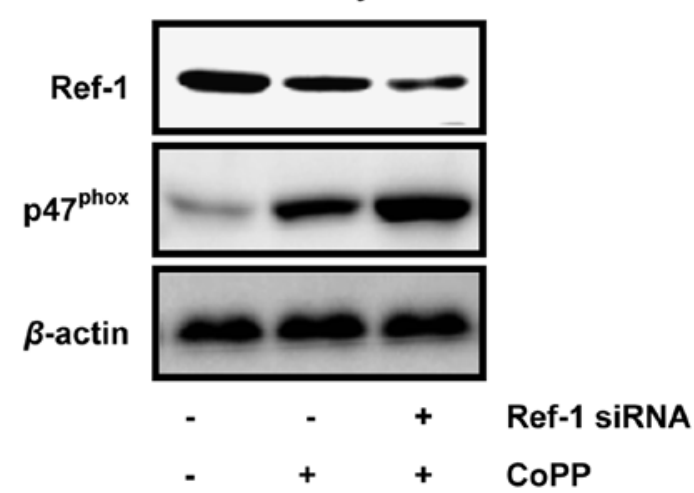

Figure 6. Involvement of Ref-1 on p47 $7^{\text {phox }}$ expression in CoPP-treated cells. (A) THP-1 cells were incubated with $100 \mu \mathrm{M}$ CoPP for up to 48 h. At the indicated time points, cytoplasmic proteins were isolated and analyzed by Western blot analysis using an antibody for p47phox . (B) Cells transfected with Ref-1 siRNA were treated with $100 \mu \mathrm{M}$ CoPP for $48 \mathrm{~h}$. Whole cell lysates were analyzed by Western blot analysis using antibodies for Ref- 1 and $\mathrm{p} 47^{\text {phox }}$. $\beta$-actin was used as an internal control.

apoptosis. Previous studies have shown that Ref-1 can regulate intracellular ROS levels through modulation of Racl-regulated NADPH oxidase $(13,14)$. Therefore, we examined whether Ref-1 modulates the activity of NADPH oxidase in CoPP-induced differentiation of THP-1 cells. As a result, a significant increase of $47^{\text {phox }}$ was observed at 24 and $48 \mathrm{~h}$ after CoPP treatment (Fig. 6A). Knockdown of Ref-1 significantly increased p47phox expression in CoPP-treated cells (Fig. 6B). This suggests that activation of NADPH oxidase in the process of monocyte differentiation may be regulated by cytoplasmic Ref-1.

\section{Discussion}

Human monocytic THP-1 cells have been widely used in the study of monocyte-macrophage differentiation because differentiated THP-1 cells behave more like native monocyte-derived macrophages than other human myeloid cell lines (15). THP-1 cells can be chemically induced to differentiate into functional macrophages when treated with various reagents including phorbol 12-myristate 13-acetate (PMA) (16), 1,25-dihydroxyvitamin D3 (17), and retinoic acid (18). It is known that the HO-1 gene is transcriptionally activated during differentiation of THP-1 cells to macrophages (19). Here, we show that CoPP induces differentiation of THP-1 cells into macrophage-like cells through activation of NADPH oxidase and that the disappearance of cytoplasmic Ref-1 may play an important role in the monocytic differentiation induced by CoPP.

Ref-1 acts as an essential regulator of the intracellular redox status. Although Ref-1 was first isolated from nuclear fractions of HeLa cells (20), numerous studies have also shown that Ref-1 has a differential cellular and subcellular expression pattern (21-23) suggesting a potential cytoplasmic role of Ref-1. Cytoplasmic Ref-1 may be required in order to maintain newly synthesized transcription factors in a reduced state while they are being translocated to the nucleus (22). Some reports suggest that decrease of Ref- 1 protein levels may be associated with differentiation $(6,8)$, although the regulatory mechanisms of Ref-1 degradation and localization are not available. Differentiation of monocytes into macrophage-like cells results in decreased amounts of Ref-1 (7).
Cytoplasmic Ref-1, alone or in concert with thioredoxin, may also modulate the activity of Rac1-regulated NADPH oxidase $(13,24)$. Furthermore, the oxidant signaling driven by NADPH oxidase can actively contribute to the monocyte differentiation into macrophages (25). Also, ROS production was significantly increased when cardiac stem cells were challenged with a pro-oxidant under Ref-1 inhibition, which led to the induction of cardiac differentiation (6). Our finding is further supported by this rationale. We demonstrated that the decrease of cytoplasmic Ref- 1 results in a significant increase in $47^{\text {phox }}$, and CoPP-induced differentiation of THP-1 cells was completely abolished by inhibition of NADPH oxidase.

We also found that CoPP increases the synthesis of ferritin protein with accumulation of HO-1 in THP-1 cells. This suggests that CoPP increases intracellular free iron through degradation of heme. When $25 \mu \mathrm{M}$ desferrioxamine (DFO), an iron chelator, was added to cultures with CoPP, adherent cells were not observed after $72 \mathrm{~h}$, and addition of external iron by treatment with $100 \mu \mathrm{M}$ ferric citrate partially recovered the inhibitory effect of DFO treatment (data not shown). Our data indicate that iron is an essential factor in CoPP-induced differentiation of THP-1 cells, suggesting a positive role for iron in monocyte differentiation. Similarly, other reports have demonstrated that iron is required for cell growth, viability, and differentiation of hematopoietic cells $(26,27)$. In contrast, it has also been reported that DFO induces THP-1 cell differentiation by modulating intracellular GSH levels (28).

In conclusion, we demonstrate that CoPP induces differentiation of human monocytic THP-1 cells into macrophage-like cells. Our data indicate that the stimulatory effect of CoPP on THP-1 cell differentiation is partially mediated by the disappearance of cytoplasmic Ref-1, which modulates the intracellular redox status through activation of NADPH oxidase.

\section{Acknowledgements}

This study was supported by the Bio-Scientific Research Grant funded by the Pusan National University (PNU-2008101-206). 


\section{References}

1. Allen RG and Venkatraj VS: Oxidants and antioxidants in development and differentiation. J Nutr 122: 631-635, 1992.

2. Sauer H, Wartenberg M and Hescheler J: Reactive oxygen species as intracellular messengers during cell growth and differentiation. Cell Physiol Biochem 11: 173-186, 2001.

3. Nakamura H, Nakamura K and Yodoi J: Redox regulation of cellular activation. Annu Rev Immunol 15: 351-369, 1997.

4. Yoo YH, Lim YJ, Park SE, Kim JM and Park YC: Overexpression of redox factor-1 negatively regulates NO synthesis and apoptosis in LPS-stimulated RAW 264.7 macrophages. FEBS Lett 556: 39-42, 2004.

5. Luo M, Delaplane S, Jiang A, Reed A,He Y, Fishel M, Nyland RL II, Borch RF, Qiao X, Georgiadis MM and Kelley MR: Role of the multifunctional DNA repair and redox signaling protein Ape1/ Ref-1 in cancer and endothelial cells: small-molecule inhibition of the redox function of Ape1. Antioxid Redox Signal 10: 1853-1867, 2008.

6. Gurusamy N, Mukherjee S, Lekli I, Bearzi C, Bardelli S and Das D: Inhibition of Ref-1 stimulates the production of reactive oxygen species and induces differentiation in adult cardiac stem cells. Antioxid Redox Signal 11: 589-599, 2009.

7. Monick MM, Carter AB and Hunninghake GW: Human alveolar macrophages are markedly deficient in REF-1 and AP-1 DNA binding activity. J Biol Chem 274: 18075-18080, 1999.

8. Chiarini LB, Freitas FG, Petrs-Silva H and Linden R: Evidence that the bifunctional redox factor/AP endonuclease Ref- 1 is an anti-apoptotic protein associated with differentiation in the developing retina. Cell Death Differ 7: 272-281, 2000.

9. Shan Y, Lambrecht RW, Donohue SE and Bonkovsky HL: Role of Bach1 and Nrf2 in up-regulation of the heme oxygenase-1 gene by cobalt protoporphyrin. FASEB J 20: E2258-E2267, 2006.

10. Galbraith RA and Kappas A: Regulation of food intake and body weight by cobalt porphyrins in animals. Proc Natl Acad Sci USA 86: 7653-7657, 1989.

11. Muhoberac BB, Hanew T, Halter S and Schenker S: A model of cytochrome $\mathrm{P}-450$-centered hepatic dysfunction in drug metabolism induced by cobalt-protoporphyrin administration. Biochem Pharmacol 38: 4103-4113, 1989.

12. Smith TJ, Drummond GS and Kappas A: Cobalt-protoporphyrin suppresses thyroid and testicular hormone concentrations in rat serum: a novel action of this synthetic heme analogue. Pharmacology 34: 9-16, 1987.

13. Angkeow P, Deshpande SS, Qi B, Liu YX, Park YC, Jeon BH, Ozaki M and Irani K: Redox factor-1: an extra-nuclear role in the regulation of endothelial oxidative stress and apoptosis. Cell Death Differ 9: 717-725, 2002.

14. Song JD, Lee SK, Kim KM, Kim JW, Kim JM, Yoo YH and Park YC: Redox factor-1 mediates NF- $\mathrm{kB}$ nuclear translocation for LPS-induced iNOS expression in murine macrophage cell line RAW 264.7. Immunology 124: 58-67, 2007.

15. Auwerx J: The human leukemia cell line, THP-1: a multifacetted model for the study of monocyte-macrophage differentiation. Experientia 47: 22-31, 1991.
16. Tsuchiya S, Kobayashi Y, Goto Y, Okumura H, Nakae S, Konno T and Tada K: Induction of maturation in cultured human monocytic leukemia cells by a phorbol diester. Cancer Res 42: 1530-1536, 1982.

17. Schwende H, Fitzke E, Ambs P and Dieter P: Differences in the state of differentiation of THP-1 cells induced by phorbol ester and 1,25-dihydroxyvitamin D3. J Leukoc Biol 59: 555-561, 1996.

18. Chen $\mathrm{Q}$ and Catharine RA: Retinoic acid regulates cell cycle progression and cell differentiation in human monocytic THP-1 cells. Exp Cell Res 297: 68-81, 2004.

19. Muraosa Y and Shibahara S: Identification of a cis-regulatory element and putative trans-acting factors responsible for 12-O-tetradecanoylphorbol 13-acetate (TPA)-mediated induction of heme oxygenase expression in myelomonocytic cell lines. Mol Cell Biol 13: 7881-7891, 1993.

20. Xanthoudakis S and Curran T: Identification and characterization of Ref-1, a nuclear protein that facilitates AP-1 DNA-binding activity. EMBO J 11: 653-665, 1992.

21. Kakolyris S, Kaklamanis L, Giatromanolaki A, Koukourakis M, Hickson ID, Barzilay G, Turley H, Lee RD, Kanavaros P, Georgoulias V, Gatter KC and Harris AL: Expression and subcellular localization of human AP endonuclease 1 (HAP1/Ref-1) protein: a basis for its role in human disease. Histopathology 33: 561-569, 1998.

22. Duguid JR, Eble JN, Wilson TM and Kelley MR: Differential cellular and subcellular expression of the human multifunctional apurinic/apyrimidinic endonuclease (APE/ref-1) DNA repair enzyme. Cancer Res 55: 6097-6102, 1995.

23. Tell G, Damante G, Caldwell D and Kelley MR: The intracellular localization of APE1/Ref-1: more than a passive phenomenon? Antioxid Redox Signal 7: 367-384, 2005.

24. Hirota K, Matsui M, Iwata S, Nishiyama A, Mori K and Yodoi J: AP-1 transcriptional activity is regulated by a direct association between thioredoxin and Ref-1. Proc Natl Acad Sci USA 94: 3633-3638, 1997.

25. Barbieri SS, Eligini S, Brambilla M, Tremoli E and Colli S: Reactive oxygen species mediate cyclooxygenase- 2 induction during monocyte to macrophage differentiation: critical role of NADPH oxidase. Cardiovasc Res 60: 187-197, 2003.

26. Gazitt Y, Reddy SV, Alcantara O, Yang J and Boldt DH: A new molecular role for iron in regulation of cell cycling and differentiation of HL-60 human leukemia cells: iron is required for transcription of p21(WAF1/CIP1) in cells induced by phorbol myristate acetate. J Cell Physiol 187: 124-135, 2001.

27. Kramer JL, Baltathakis I, Alcantara OS and Boldt DH Differentiation of functional dendritic cells and macrophages from human peripheral blood monocyte precursors is dependent on expression of p21 (WAF1/CIP1) and requires iron. Br J Haematol 117: 727-734, 2002

28. Seo GS, Lee SH, Choi SC, Choi EY, Oh HM, Choi EJ, Park DS, Kim SW, Kim TH, Nah YH, Kim S, Kim SH, You SH and Jun CD: Iron chelator induces THP-1 cell differentiation potentially by modulating intracellular glutathione levels. Free Radic Biol Med 40: 1502-1512, 2006. 